Lie Theory and Its Applications in Physics VII

eds. H.-D. Doebner and V.K. Dobrev, Heron Press, Sofia, 2008

\title{
Asymmetric Wormholes via Electrically Charged Lightlike Branes
}

\author{
E. Guendelman*1, A. Kaganovich ${ }^{\dagger 1}$, E. Nissimov ${ }^{\ddagger 2}$, \\ S. Pacheva ${ }^{\S 2}$ \\ ${ }^{1}$ Department of Physics, Ben-Gurion University of the Negev, P.O.Box 653, \\ IL-84105 Beer-Sheva, Israel \\ ${ }^{2}$ Institute for Nuclear Research and Nuclear Energy, Bulgarian Academy of \\ Sciences, Boul. Tsarigradsko Chausee 72, BG-1784 Sofia, Bulgaria
}

\begin{abstract}
We consider a self-consistent Einstein-Maxwell-Kalb-Ramond system in the bulk $D=4$ space-time interacting with a variable-tension electrically charged lightlike brane. The latter serves both as a material and charge source for gravity and electromagnetism, as well as it dynamically generates a bulk space varying cosmological constant. We find an asymmetric wormhole solution describing two "universes" with different spherically symmetric black-hole-type geometries connected through a "throat" occupied by the lightlike brane. The electrically neutral "left universe" comprises the exterior region of Schwarzschild-de-Sitter (or pure Schwarzschild) space-time above the inner (Schwarzschild-type) horizon, whereas the electrically charged "right universe" consists of the exterior Reissner-Nordström (or Reissner-Nordström-de-Sitter) black hole region beyond the outer Reissner-Nordström horizon. All physical parameters of the wormhole are uniquely determined by two free parameters - the electric charge and Kalb-Ramond coupling of the lightlike brane.
\end{abstract}

\section{Introduction}

Lightlike brane ( $L L$-branes for short) play an important role in general relativity as they enter the description of various physically important cosmological and astrophysical phenomena such as: (i) impulsive lightlike signals arising in cataclysmic astrophysical events [1]; (ii) the "membrane paradigm" [2] of black hole physics; (iii) the thin-wall approach to domain walls coupled to gravity [3-5].

\footnotetext{
*guendel@bgu.ac.il

†alexk@bgu.ac.il

§nissimov@inrne.bas.bg, svetlana@inrne.bas.bg
} 
More recently, $L L$-branes became significant also in the context of modern nonperturbative string theory, in particular, as the so called $H$-branes describing quantum horizons (black hole and cosmological) [6], as Penrose limits of baryonic $D$-branes [7], etc (see also Refs. [8]).

In the pioneering papers [3-5] LL-branes in the context of gravity and cosmology have been extensively studied from a phenomenological point of view, i.e., by introducing them without specifying the Lagrangian dynamics from which they may originate ${ }^{1}$. On the other hand, we have proposed in a series of recent papers [10-13] a new class of concise Lagrangian actions, providing a derivation from first principles of the $L L$-brane dynamics.

There are several characteristic features of $L L$-branes which drastically distinguish them from ordinary Nambu-Goto branes:

(i) They describe intrinsically lightlike modes, whereas Nambu-Goto branes describe massive ones.

(ii) The tension of the LL-brane arises as an additional dynamical degree of freedom, whereas Nambu-Goto brane tension is a given ad hoc constant. The latter characteristic feature significantly distinguishes our $L L$-brane models from the previously proposed tensionless $p$-branes (for a review, see Ref. [14]) which rather resemble a $p$-dimensional continuous distribution of massless point-particles.

(iii) Consistency of $L L$-brane dynamics in a spherically or axially symmetric gravitational background of codimension one requires the presence of an event horizon which is automatically occupied by the $L L$-brane ("horizon straddling" according to the terminology of Ref. [4]).

(iv) When the $L L$-brane moves as a test brane in spherically or axially symmetric gravitational backgrounds its dynamical tension exhibits exponential "inflation/deflation" time behaviour [11] - an effect similar to the "mass inflation" effect around black hole horizons [15].

In a series of recent papers $[12,13,16]$ we have explored the novel possibility of employing $L L$-branes as natural self-consistent gravitational sources for wormhole space-times, in other words, generating wormhole solutions in self-consistent bulk gravity-matter systems coupled to $L L$-branes through dynamically derived world-volume $L L$-brane stress energy tensors. For a review of wormhole space-times, see Refs. [17, 18].

The possibility of a "wormhole space-time" was first hinted at in the work of Einstein and Rosen [19], where they considered matching at the horizon of two identical copies of the exterior Schwarzschild space-time region (subsequently called Einstein-Rosen "bridge"). The original Einstein-Rosen "bridge" manifold appears as a particular case of the construction of spherically symmetric wormholes produced by $L L$-branes as gravitational sources (Refs. [13, 16]; see also Section 5 below). The main lesson here is that consistency of Einstein equations of motion yielding the original Einstein-Rosen "bridge" as well-defined solution necessarily requires the presence of $L L$-brane energy-momentum tensor

\footnotetext{
${ }^{1}$ In a more recent paper [9] brane actions in terms of their pertinent extrinsic geometry have been proposed which generically describe non-lightlike branes, whereas the lightlike branes are treated as a limiting case.
} 
as a source on the right hand side. Thus, the introduction of LL-brane coupling to gravity brings the original Einstein-Rosen construction in Ref. [19] to a consistent completion ${ }^{2}$.

More complicated examples of spherically and axially symmetric wormholes with Reissner-Nordström and rotating cylindrical geometry, respectively, have also been presented in Refs. [12,13]. Namely, two copies of the outer space-time region of a Reissner-Nordström or rotating cylindrical black hole, respectively, are matched via $L L$-brane along what used to be the outer horizon of the respective full black hole space-time manifold. In this way we obtain a wormhole solution which combines the features of the Einstein-Rosen "bridge" on the one hand (with wormhole throat at horizon), and the features of MisnerWheeler wormholes [21], i.e., exhibiting the so called "charge without charge" phenomenon $^{1}$, on the other hand.

In the present note the results of Refs. $[12,13]$ will be extended to the case of asymmetric wormholes, describing two "universes" with different spherically symmetric geometries of black hole type connected via a "throat" occupied by the pertinent gravitational source - an electrically charged LL-brane. As a result of the well-defined world-volume $L L$-brane dynamics coupled self-consistently to gravity and bulk space-time gauge fields, it creates a "left universe" with Schwarzschild-de-Sitter geometry where the cosmological constant is dynamically generated, and a "right universe" with Reissner-Nordström geometry with dynamically generated Coulomb field-strength. Similarly, the LL-brane can dynamically generate a non-zero cosmological constant in the "right universe", in which case it connects a purely Schwarzschild "left universe" with a ReissnerNordström-de-Sitter "right universe".

The presentation of the material goes as follows. In Section 2 we briefly review the reparametrization-invariant world-volume Lagrangian formulation of LL-branes in both the Polyakov-type and Nambu-Goto-type forms. In Section 3 we briefly describe the main properties of $L L$-brane dynamics in spherically symmetric gravitational backgrounds stressing particularly on the "horizon straddling" phenomenon and the dynamical cosmological constant generation. Section 4 contains our principal result - the explicit construction of

\footnotetext{
${ }^{2}$ Let us particularly emphasize that here and in what follows we consider the Einstein-Rosen "bridge" in its original formulation in Ref. [19] as a four-dimensional space-time manifold consisting of two copies of the exterior Schwarzschild space-time region matched along the horizon. On the other hand, the nomenclature of "Einstein-Rosen bridge" in several standard textbooks (e.g. Ref. [20]) uses the Kruskal-Szekeres manifold. The latter notion of "Einstein-Rosen bridge" is not equivalent to the original construction in Ref. [19]. Namely, the two regions in Kruskal-Szekeres space-time corresponding to the outer Schwarzschild space-time region $(r>2 m)$ and labeled $(I)$ and $(I I I)$ in Refs. [20] are generally disconnected and share only a two-sphere (the angular part) as a common border ( $U=0, V=0$ in Kruskal-Szekeres coordinates), whereas in the original Einstein-Rosen "bridge" construction the boundary between the two identical copies of the outer Schwarzschild space-time region $(r>2 m)$ is a three-dimensional hypersurface $(r=2 m)$.

${ }^{1}$ Misner and Wheeler [21] realized that wormholes connecting two asymptotically flat space times provide the possibility of "charge without charge", i.e., electromagnetically non-trivial solutions where the lines of force of the electric field flow from one universe to the other without a source and giving the impression of being positively charged in one universe and negatively charged in the other universe.
} 
an asymmetric wormhole solution of self-consistent Einstein-Maxwell-KalbRamond system interacting with an electrically charged LL-brane. The wormhole space-time consists of two "universes" with different spherically symmetric geometries connected through a "throat" occupied by the LL-brane: (a) electrically neutral "left universe" comprising the exterior region of Schwarzschildde-Sitter (or pure Schwarzschild) space-time above the inner (Schwarzschildtype) horizon; (b) electrically charged "right universe" consisting of the exterior Reissner-Nordström (or Reissner-Nordström-de-Sitter) black hole region beyond the outer Reissner-Nordström horizon. In Section 5 we briefly consider the simple special case of the above construction with vanishing charge and Kalb-Ramond coupling of the LL-branes. It consistently describes the famous Einstein-Rosen "bridge" wormhole solution [19]. On the way we explain the crucial role of the presence of the $L L$-brane gravitational source producing the Einstein-Rosen "bridge" - an observation missing in the original classic paper [19].

\section{Einstein-Maxwell-Kalb-Ramond System Interacting With Light- like Brane: Lagrangian Formulation}

Self-consistent bulk Einstein-Maxwell-Kalb-Ramond system coupled to a charged codimension-one lightlike $p$-brane (i.e., $D=(p+1)+1)$ is described by the following action:

$$
S=\int d^{D} x \sqrt{-G}\left[\frac{R(G)}{16 \pi}-\frac{1}{4} \mathcal{F}_{\mu \nu} \mathcal{F}^{\mu \nu}-\frac{1}{D ! 2} \mathcal{F}_{\mu_{1} \ldots \mu_{D}} \mathcal{F}^{\mu_{1} \ldots \mu_{D}}\right]+\widetilde{S}_{\mathrm{LL}} .
$$

Here $\mathcal{F}_{\mu \nu}=\partial_{\mu} \mathcal{A}_{\nu}-\partial_{\nu} \mathcal{A}_{\mu}$ and

$$
\mathcal{F}_{\mu_{1} \ldots \mu_{D}}=D \partial_{\left[\mu_{1}\right.} \mathcal{A}_{\left.\mu_{2} \ldots \mu_{D}\right]}=\mathcal{F} \sqrt{-G} \varepsilon_{\mu_{1} \ldots \mu_{D}}
$$

are the field-strengths of the electromagnetic $\mathcal{A}_{\mu}$ and Kalb-Ramond $\mathcal{A}_{\mu_{1} \ldots \mu_{D-1}}$ gauge potentials [22]. The last term on the r.h.s. of (1) indicates the reparametrization invariant world-volume action of the $L L$-brane coupled to the bulk gauge fields, proposed in our previous papers [10-12]:

$$
\begin{array}{r}
\widetilde{S}_{\mathrm{LL}}=S_{\mathrm{LL}}-q \int d^{p+1} \sigma \varepsilon^{a b_{1} \ldots b_{p}} F_{b_{1} \ldots b_{p}} \partial_{a} X^{\mu} \mathcal{A}_{\mu} \\
-\frac{\beta}{(p+1) !} \int d^{p+1} \sigma \varepsilon^{a_{1} \ldots a_{p+1}} \partial_{a_{1}} X^{\mu_{1}} \ldots \partial_{a_{p+1}} X^{\mu_{p+1}} \mathcal{A}_{\mu_{1} \ldots \mu_{p+1}}
\end{array}
$$

where:

$$
S_{\mathrm{LL}}=\int d^{p+1} \sigma \Phi\left[-\frac{1}{2} \gamma^{a b} g_{a b}+L\left(F^{2}\right)\right] .
$$

In Eqs.(3)-(4) the following notions and notations are used:

- $\Phi$ is alternative non-Riemannian integration measure density (volume 
form) on the $p$-brane world-volume manifold:

$$
\begin{gathered}
\Phi \equiv \frac{1}{(p+1) !} \varepsilon^{a_{1} \ldots a_{p+1}} H_{a_{1} \ldots a_{p+1}}(B), \\
H_{a_{1} \ldots a_{p+1}}(B)=(p+1) \partial_{\left[a_{1}\right.} B_{\left.a_{2} \ldots a_{p+1}\right]},
\end{gathered}
$$

instead of the usual $\sqrt{-\gamma}$. Here $\varepsilon^{a_{1} \ldots a_{p+1}}$ is the alternating symbol $\left(\varepsilon^{01 \ldots p}=1\right), \gamma_{a b}(a, b=0,1, \ldots, p)$ indicates the intrinsic Riemannian metric on the world-volume, and $\gamma=\operatorname{det}\left\|\gamma_{a b}\right\| . H_{a_{1} \ldots a_{p+1}}(B)$ denotes the field-strength of an auxiliary world-volume antisymmetric tensor gauge field $B_{a_{1} \ldots a_{p}}$ of rank $p$. As a special case one can build $H_{a_{1} \ldots a_{p+1}}$ (6) in terms of $p+1$ auxiliary world-volume scalar fields $\left\{\varphi^{I}\right\}_{I=1}^{p+1}$ :

$$
H_{a_{1} \ldots a_{p+1}}=\varepsilon_{I_{1} \ldots I_{p+1}} \partial_{a_{1}} \varphi^{I_{1}} \ldots \partial_{a_{p+1}} \varphi^{I_{p+1}} .
$$

Note that $\gamma_{a b}$ is independent of the auxiliary world-volume fields $B_{a_{1} \ldots a_{p}}$ or $\varphi^{I}$. The alternative non-Riemannian volume form (5) has been first introduced in the context of modified standard (non-lightlike) string and $p$-brane models in Refs. [23].

- $X^{\mu}(\sigma)$ are the $p$-brane embedding coordinates in the bulk $D$-dimensional space time with bulk Riemannian metric $G_{\mu \nu}(X)$ with $\mu, \nu=$ $0,1, \ldots, D-1 ;(\sigma) \equiv\left(\sigma^{0} \equiv \tau, \sigma^{i}\right)$ with $i=1, \ldots, p ; \partial_{a} \equiv \frac{\partial}{\partial \sigma^{a}}$.

- $g_{a b}$ is the induced metric on world-volume:

$$
g_{a b} \equiv \partial_{a} X^{\mu} \partial_{b} X^{\nu} G_{\mu \nu}(X),
$$

which becomes singular on-shell (manifestation of the lightlike nature, $\mathrm{cf}$. second Eq.(25) below).

- $L\left(F^{2}\right)$ is the Lagrangian density of another auxiliary $(p-1)$-rank antisymmetric tensor gauge field $A_{a_{1} \ldots a_{p-1}}$ on the world-volume with $p$-rank field-strength and its dual:

$$
F_{a_{1} \ldots a_{p}}=p \partial_{\left[a_{1}\right.} A_{\left.a_{2} \ldots a_{p}\right]} \quad, \quad F^{* a}=\frac{1}{p !} \frac{\varepsilon^{a a_{1} \ldots a_{p}}}{\sqrt{-\gamma}} F_{a_{1} \ldots a_{p}} .
$$

$L\left(F^{2}\right)$ is arbitrary function of $F^{2}$ with the short-hand notation:

$$
F^{2} \equiv F_{a_{1} \ldots a_{p}} F_{b_{1} \ldots b_{p}} \gamma^{a_{1} b_{1}} \ldots \gamma^{a_{p} b_{p}}
$$

Rewriting the action (4) in the following equivalent form:

$$
S=-\int d^{p+1} \sigma \chi \sqrt{-\gamma}\left[\frac{1}{2} \gamma^{a b} \partial_{a} X^{\mu} \partial_{b} X^{\nu} G_{\mu \nu}(X)-L\left(F^{2}\right)\right], \chi \equiv \frac{\Phi}{\sqrt{-\gamma}}
$$

with $\Phi$ the same as in (5), we find that the composite field $\chi$ plays the role of a dynamical (variable) brane tension. The notion of dynamical brane tension has previously appeared in different contexts in Refs. [24]. 
Let us also remark that, as it has been shown in Refs. [12,28], the LL-brane equations of motion corresponding to the Polyakov-type action (4) (or (11)) can be equivalently obtained from the following dual Nambu-Goto-type action:

$$
S_{\mathrm{NG}}=-\int d^{p+1} \sigma T \sqrt{\left|\operatorname{det}\left\|g_{a b}-\epsilon \frac{1}{T^{2}} \partial_{a} u \partial_{b} u\right\|\right|} \quad, \quad \epsilon= \pm 1 .
$$

Here $T$ is dynamical tension simply proportional to the dynamical tension in the Polyakov-type formulation (4) ( $\left.T \sim \chi=\frac{\Phi}{\sqrt{-\gamma}}\right)$, and $u$ denotes the dual potential w.r.t. $A_{a_{1} \ldots a_{p-1}}$ :

$$
F_{a}^{*}(A)=\operatorname{const} \frac{1}{\chi} \partial_{a} u
$$

It what follows we will consider the original Polyakov-type action (4).

The pertinent Einstein-Maxwell-Kalb-Ramond equations of motion derived from the action (1) read:

$$
\begin{gathered}
R_{\mu \nu}-\frac{1}{2} G_{\mu \nu} R=8 \pi\left(T_{\mu \nu}^{(E M)}+T_{\mu \nu}^{(K R)}+T_{\mu \nu}^{(\text {brane })}\right) \\
\partial_{\nu}\left(\sqrt{-G} \mathcal{F}^{\mu \nu}\right)+q \int d^{p+1} \sigma \delta^{(D)}(x-X(\sigma)) \varepsilon^{a b_{1} \ldots b_{p}} F_{b_{1} \ldots b_{p}} \partial_{a} X^{\mu}=0 \\
\varepsilon^{\nu \mu_{1} \ldots \mu_{p+1}} \partial_{\nu} \mathcal{F}- \\
\beta \int d^{p+1} \sigma \delta^{(D)}(x-X(\sigma)) \varepsilon^{a_{1} \ldots a_{p+1}} \partial_{a_{1}} X^{\mu_{1}} \ldots \partial_{a_{p+1}} X^{\mu_{p+1}}=0
\end{gathered}
$$

where in the last equation we have used relation (2). The explicit form of the energy-momentum tensors read:

$$
\begin{gathered}
T_{\mu \nu}^{(E M)}=\mathcal{F}_{\mu \kappa} \mathcal{F}_{\nu \lambda} G^{\kappa \lambda}-G_{\mu \nu} \frac{1}{4} \mathcal{F}_{\rho \kappa} \mathcal{F}_{\sigma \lambda} G^{\rho \sigma} G^{\kappa \lambda} \\
T_{\mu \nu}^{(K R)}=\frac{1}{(D-1) !}\left[\mathcal{F}_{\mu \lambda_{1} \ldots \lambda_{D-1}} \mathcal{F}_{\nu}{ }^{\lambda_{1} \ldots \lambda_{D-1}}-\frac{1}{2 D} G_{\mu \nu} \mathcal{F}_{\lambda_{1} \ldots \lambda_{D}} \mathcal{F}^{\lambda_{1} \ldots \lambda_{D}}\right] \\
=-\frac{1}{2} \mathcal{F}^{2} G_{\mu \nu} \\
T_{\mu \nu}^{(\text {brane })}=-G_{\mu \kappa} G_{\nu \lambda} \int d^{p+1} \sigma \frac{\delta^{(D)}(x-X(\sigma))}{\sqrt{-G}} \chi \sqrt{-\gamma} \gamma^{a b} \partial_{a} X^{\kappa} \partial_{b} X^{\lambda},
\end{gathered}
$$

where the brane stress-energy tensor is straightforwardly derived from the world-volume action (4) (or, equivalently, (11); recall $\chi \equiv \frac{\Phi}{\sqrt{-\gamma}}$ is the variable brane tension).

Eqs.(15)-(16) show that:

(i) the $L L$-brane is charged source for the bulk electromagnetism;

(ii) the $L L$-brane uniquely determines the value of $\mathcal{F}^{2}$ in Eq.(18) through its coupling to the bulk Kalb-Ramond gauge field (Eq.(16)) which implies dynamical generation of bulk cosmological constant $\Lambda=4 \pi \mathcal{F}^{2}$. 


\section{Lightlike Brane Dynamics in Spherically Symmetric Gravita- tional Backgrounds}

The equations of motion of the $L L$-brane are discussed at length in our previous papers [10-13]. Their explicit form reads:

$$
\partial_{a}\left[\frac{1}{2} \gamma^{c d} g_{c d}-L\left(F^{2}\right)\right]=0 \quad \longrightarrow \quad \frac{1}{2} \gamma^{c d} g_{c d}-L\left(F^{2}\right)=M,
$$

where $M$ is an arbitrary integration constant;

$$
\frac{1}{2} g_{a b}-F^{2} L^{\prime}\left(F^{2}\right)\left[\gamma_{a b}-\frac{F_{a}^{*} F_{b}^{*}}{F^{* 2}}\right]=0,
$$

where $F^{* a}$ is the dual world-volume field strength (9);

$$
\begin{array}{r}
\partial_{[a}\left(F_{b]}^{*} \chi L^{\prime}\left(F^{2}\right)\right)+\frac{q}{4} \partial_{a} X^{\mu} \partial_{b} X^{\nu} \mathcal{F}_{\mu \nu}=0 ; \\
\partial_{a}\left(\chi \sqrt{-\gamma} \gamma^{a b} \partial_{b} X^{\mu}\right)+\chi \sqrt{-\gamma} \gamma^{a b} \partial_{a} X^{\nu} \partial_{b} X^{\lambda} \Gamma_{\nu \lambda}^{\mu} \\
-q \varepsilon^{a b_{1} \ldots b_{p}} F_{b_{1} \ldots b_{p}} \partial_{a} X^{\nu} \mathcal{F}_{\lambda \nu} G^{\lambda \mu} \\
-\frac{\beta}{(p+1) !} \varepsilon^{a_{1} \ldots a_{p+1}} \partial_{a_{1}} X^{\mu_{1}} \ldots \partial_{a_{p+1}} X^{\mu_{p+1}} \mathcal{F}_{\lambda \mu_{1} \ldots \mu_{p+1}} G^{\lambda \mu}=0 .
\end{array}
$$

Here $\chi$ is the dynamical brane tension as in $(11), \mathcal{F}_{\lambda \mu_{1} \ldots \mu_{p+1}}$ is the KalbRamond field-strength (2),

$$
\Gamma_{\nu \lambda}^{\mu}=\frac{1}{2} G^{\mu \kappa}\left(\partial_{\nu} G_{\kappa \lambda}+\partial_{\lambda} G_{\kappa \nu}-\partial_{\kappa} G_{\nu \lambda}\right)
$$

is the Christoffel connection for the external metric, and $L^{\prime}\left(F^{2}\right)$ denotes derivative of $L\left(F^{2}\right)$ w.r.t. the argument $F^{2}$.

Eqs.(20)-(21) imply the following important consequences:

$$
F^{2}=F^{2}(M)=\mathrm{const} \quad, \quad g_{a b} F^{* b}=0,
$$

where the second equation is the manifestation of the lightlike nature of the $p$-brane model (4), i.e., the tangent vector to the world-volume $F^{* a} \partial_{a} X^{\mu}$ is lightlike w.r.t. metric of the embedding space-time.

World-volume reparametrization invariance allows us to introduce the standard synchronous gauge-fixing conditions:

$$
\gamma^{0 i}=0(i=1, \ldots, p), \gamma^{00}=-1 .
$$

Also, we will use a natural ansatz for the "electric" part of the auxiliary worldvolume gauge field-strength (9):

$$
F^{* i}=0(i=1, \ldots, p) \quad, \quad \text { i.e. } F_{0 i_{1} \ldots i_{p-1}}=0,
$$

meaning that we choose the lightlike direction in Eq.(25) to coincide with the brane proper-time direction on the world-volume $\left(F^{* a} \partial_{a} \sim \partial_{\tau}\right)$. The Bianchi 
identity $\left(\nabla_{a} F^{* a}=0\right)$ together with (26)-(27) and the definition for the dual field-strength in (9) imply:

$$
\partial_{0} \gamma^{(p)}=0 \quad \text { where } \gamma^{(p)} \equiv \operatorname{det}\left\|\gamma_{i j}\right\|
$$

Taking into account (26)-(27), Eqs.(21) acquire the following gauge-fixed form (recall definition of the induced metric $\left.g_{a b}(8)\right)$ :

$$
g_{00} \equiv \dot{X}^{\mu} G_{\mu \nu} \dot{X}^{\nu}=0 \quad, \quad g_{0 i}=0 \quad, \quad g_{i j}-2 a_{0} \gamma_{i j}=0
$$

where $a_{0}$ is a $M$-dependent constant:

$$
\left.a_{0} \equiv F^{2} L^{\prime}\left(F^{2}\right)\right|_{F^{2}=F^{2}(M)}
$$

Eqs.(29) are analogs of the Virasoro constraints in standard string theory.

In what follows we will be interested in static spherically symmetric solutions of Einstein-Maxwell-Kalb-Ramond equations (14)-(16). The generic form of spherically symmetric metric in Eddington-Finkelstein coordinates [25] reads:

$$
d s^{2}=-A(r) d v^{2}+2 d v d r+C(r) h_{i j}(\theta) d \theta^{i} d \theta^{j}
$$

where $h_{i j}$ indicates the standard metric on $S^{p}$. We will consider the simplest ansatz for the LL-brane embedding coordinates:

$$
X^{0} \equiv v=\tau \quad, \quad X^{1} \equiv r=r(\tau) \quad, \quad X^{i} \equiv \theta^{i}=\sigma^{i} \quad(i=1, \ldots, p)
$$

Now, the LL-brane equations (29) together with (28) yield:

$$
-A(r)+2 \dot{r}=0 \quad, \quad \partial_{\tau} C=\left.\dot{r} \quad \partial_{r} C\right|_{r=r(\tau)}=0,
$$

implying:

$$
\dot{r}=0 \rightarrow r=r_{0}=\text { const } \quad, \quad A\left(r_{0}\right)=0 .
$$

Eq.(34) tells us that consistency of $L L$-brane dynamics in a spherically symmetric gravitational background of codimension one requires the latter to possess a horizon (at some $r=r_{0}$ ), which is automatically occupied by the LL-brane ("horizon straddling" according to the terminology of Ref. [4]). Similar property - "horizon straddling", has been found also for LL-branes moving in rotating axially symmetric (Kerr or Kerr-Newman) and rotating cylindrically symmetric black hole backgrounds [12,13].

Next, the Maxwell coupling of the LL-brane produces via Eq.(15) static Coulomb field in the outer region beyond the horizon (for $r>r_{0}$ ). Namely, inserting in Eq.(15) the embedding ansatz (32) together with (34) and accounting for (26)-(29) we obtain;

$$
\partial_{r}\left(C^{p / 2}(r) \mathcal{F}_{v r}(r)\right)-q \frac{\sqrt{p ! F^{2}}}{\left(2 a_{0}\right)^{p / 2}} C^{p / 2}\left(r_{0}\right) \delta\left(r-r_{0}\right)=0
$$

which yields for the Maxwell field-strength:

$$
\mathcal{F}_{v r}(r)=\left(\frac{C\left(r_{0}\right)}{C(r)}\right)^{p / 2} \frac{q \sqrt{p ! F^{2}}}{\left(2 a_{0}\right)^{p / 2}} \theta\left(r-r_{0}\right) .
$$


Using again the embedding ansatz (32) together with (34) as well as (26)(29), the Kalb-Ramond equations of motion (16) reduce to:

$$
\begin{aligned}
\partial_{r} \mathcal{F}+\beta \delta\left(r-r_{0}\right)=0 \rightarrow & \mathcal{F}=\mathcal{F}_{(+)} \theta\left(r-r_{0}\right)+\mathcal{F}_{(-)} \theta\left(r_{0}-r\right) \\
& \mathcal{F}_{( \pm)}=\mathrm{const} \quad, \quad \mathcal{F}_{(-)}-\mathcal{F}_{(+)}=\beta
\end{aligned}
$$

Therefore, a space-time varying non-negative cosmological constant is dynamically generated in both exterior and interior regions w.r.t. the horizon at $r=r_{0}$ (cf. Eq.(18)):

$$
\Lambda_{( \pm)}=4 \pi \mathcal{F}_{( \pm)}^{2}
$$

Finally, it remains to consider the second order (w.r.t. proper time derivative) $X^{\mu}$ equations of motion (23). Upon inserting the embedding ansatz (32) together with (34) and taking into account (29), (36) and (38), we find that the only non-trivial equations is for $\mu=v$. Before proceeding let us note that the "force" terms in the $X^{\mu}$ equations of motion (23) (the geodesic ones containing the Christoffel connection coefficients as well as those coming from the LL-brane coupling to the bulk Maxwell and Kalb-Ramond gauge fields) contain discontinuities across the horizon occupied by the $L L$-brane. The discontinuity problem is resolved following the approach in Ref. [3] (see also the regularization approach in Ref. [26], Appendix A) by taking mean values of the "force" terms across the discontinuity at $r=r_{0}$. Thus, we obtain from Eq.(23) with $\mu=v$ :

$$
\begin{aligned}
\partial_{\tau} \chi+\chi\left[\frac { 1 } { 4 } \left(\partial_{r} A_{(+)}\right.\right. & \left.\left.+\partial_{r} A_{(-)}\right)+\frac{1}{2} p a_{0}\left(\partial_{r} \ln C_{(+)}+\partial_{r} \ln C_{(-)}\right)\right]_{r=r_{0}} \\
+ & \frac{1}{2}\left[-q^{2} \frac{p ! F^{2}}{\left(2 a_{0}\right)^{p / 2}}+\beta\left(2 a_{0}\right)^{p / 2}\left(\mathcal{F}_{(-)}+\mathcal{F}_{(+)}\right)\right]=0 .
\end{aligned}
$$

\section{Asymmetric Wormhole Solution}

The LL-brane energy-momentum tensor (19) on the r.h.s. of the Einstein equations of motion (14), upon inserting the expressions for $X^{\mu}(\sigma)$ from (32) and (34), and taking into account the gauge-fixing conditions (26) and the ansatz (27), acquires the form:

$$
T_{(\text {brane })}^{\mu \nu}=S^{\mu \nu} \delta\left(r-r_{0}\right)
$$

with surface energy-momentum tensor:

$$
S^{\mu \nu} \equiv \frac{\chi}{\left(2 a_{0}\right)^{p / 2}}\left[\partial_{\tau} X^{\mu} \partial_{\tau} X^{\nu}-2 a_{0} G^{i j} \partial_{i} X^{\mu} \partial_{j} X^{\nu}\right]_{v=\tau, r=r_{0}, \theta^{i}=\sigma^{i}} .
$$

Here $a_{0}$ is the integration constant parameter appearing in the $L L$-brane dynamics (30) and $G_{i j}=C(r) h_{i j}(\theta)$. For the non-zero components of $S_{\mu \nu}$ (with lower indices) and its trace we find:

$$
S_{r r}=\frac{\chi}{\left(2 a_{0}\right)^{p / 2}} \quad, \quad S_{i j}=-\frac{\chi}{\left(2 a_{0}\right)^{p / 2-1}} G_{i j} \quad, \quad S_{\lambda}^{\lambda}=-\frac{p \chi}{\left(2 a_{0}\right)^{p / 2-1}}
$$


The solution of the other bulk space-time equations of motion (the Maxwell (15) and Kalb-Ramond (16)) with spherically symmetric geometry have already been given in the previous Section, see Eqs.(35)-(38).

For the sake of simplicity we will consider in what follows the case of $D=$ 4-dimensional bulk space-time and, correspondingly, $p=2$ for the $L L$-brane. The generalization to arbitrary $D$ is straightforward. For further simplification of the numerical constant factors we will choose the following specific ("wrongsign" Maxwell) form for the Lagrangian of the auxiliary non-dynamical worldvolume gauge field (cf. Eqs.(9)-(10)):

$$
L\left(F^{2}\right)=\frac{1}{4} F^{2} \rightarrow a_{0}=M,
$$

where again $a_{0}$ is the constant defined in (30) and $M$ denotes the original integration constant in Eqs.(20).

We will show that there exists an asymmetric wormhole solution of the Einstein equations of motion (14) with $L L$-brane energy-momentum tensor on the r.h.s. given by (42)-(43) - systematically derived from the reparametrization invariant $L L$-brane world-volume action (4), which describes two "universes" with different spherically symmetric geometries (31) matched along a $L L$-brane. More specifically, this solution describes an overall space-time manifold containing two separate spherically symmetric space-time regions:

(i) a "left universe" consisting of the exterior region of Schwarzschild-deSitter space-time above the inner (Schwarzschild-type) horizon, i.e., with metric (31) where $C(r)=r^{2}$ and:

$$
\begin{array}{r}
A(r) \equiv A_{(-)}(r)=1-\frac{2 m_{1}}{r}-K r^{2} \quad \text { for } r>r_{0}, \\
A_{(-)}\left(r_{0}\right)=0 \quad,\left.\quad \partial_{r} A_{(-)}\right|_{r=r_{0}}>0 ;
\end{array}
$$

(ii) a "right universe" comprising the exterior Reissner-Nordström black hole region beyond the outer Reissner-Nordström horizon with metric (31) where $C(r)=r^{2}$ and:

$$
\begin{array}{r}
A(r) \equiv A_{(+)}(r)=1-\frac{2 m_{2}}{r}+\frac{Q^{2}}{r^{2}} \quad \text { for } r>r_{0}, \\
A_{(+)}\left(r_{0}\right)=0 \quad,\left.\quad \partial_{r} A_{(+)}\right|_{r=r_{0}}>0 .
\end{array}
$$

The "throat" connecting the above two "universes" with metrics (45) and (47) is the lightlike world-volume hypersurface of the $L L$-brane which is located on the common horizon $\left(r=r_{0}\right)$ of both "universes" - a Schwarzschild-type horizon from the "left universe" side (46) and an outer Reissner-Nordström horizon from the "right universe" side (48). As already pointed out above (Eqs.(33)(34)), the common horizon at $r=r_{0}$ is automatically occupied by the $L L$-brane ("horizon straddling") as a result of its world-volume dynamics.

The asymmetric wormhole solution under consideration is explicitly given in Eddington-Finkelstein-type coordinates as:

$$
d s^{2}=-\widetilde{A}(\eta) d v^{2}+2 d v d \eta+\widetilde{r}^{2}(\eta)\left[d \theta^{2}+\sin ^{2} \theta d \varphi^{2}\right],
$$


where the original radial coordinate $r(r>0)$ is replaced by $\eta(-\infty<\eta<\infty)$ upon substituting:

$$
r \rightarrow \widetilde{r}(\eta)=r_{0}+|\eta|
$$

with $r_{0}$ - the common horizon of (45) and (47) as follows:

$$
\begin{gathered}
\widetilde{A}(\eta) \equiv A_{(-)}(\widetilde{r}(\eta))=1-\frac{2 m_{1}}{\widetilde{r}(\eta)}-K \widetilde{r}^{2}(\eta) \quad, K \equiv \frac{4 \pi}{3} \beta^{2}, \text { for } \eta<0, \\
\widetilde{A}(\eta) \equiv A_{(+)}(\widetilde{r}(\eta))=1-\frac{2 m_{2}}{\widetilde{r}(\eta)}+\frac{Q^{2}}{\widetilde{r}^{2}(\eta)} \quad, \quad Q^{2} \equiv \frac{8 \pi}{a_{0}} q^{2} r_{0}^{4}, \text { for } \eta>0 .
\end{gathered}
$$

The new radial-like coordinate $\eta$ describes a continuous interpolation between the left $(\eta<0)$ and the right $(\eta>0)$ "universes" through the "throat" at $\eta=$ 0. As shown in Eqs.(37)-(39), the LL-brane through its coupling to the bulk Kalb-Ramond field (cf. (3) and (16)) dynamically generates space-time varying non-negative cosmological constant with a jump across the horizon $\left(r=r_{0}\right)$. In the present case this yields dynamically generated de Sitter parameter $K=$ $\frac{1}{3} \Lambda_{(-)} \equiv \frac{4 \pi}{3} \mathcal{F}_{(-)}^{2}=\frac{4 \pi}{3} \beta^{2}$ in the "left universe" as given in (51), whereas $\mathcal{F}_{(+)}=0$. On the other hand, the surface charge density $q$ of the $L L$-brane (cf. (15) and (35)) explicitly determines the non-zero Coulomb field-strength in the "right universe" with $Q^{2}$ as given in (52).

Now, substituting the metric (49) with (51)-(52) into the Einstein equations (14) and taking into account that outside the "throat" $(\eta=0)$ it obviously solves the "vacuum" equations (with the $L L$-brane absent), the only non-trivial $\delta$-function contribution on the 1.h.s. of (14) arises because of non-smoothness of the metric (49) with (51)-(52) at $\eta=0$ (it is continuous but not differentiable there). Thus, inserting the world-volume Lagrangian-derived expression (41)(42) for the $L L$-brane stress-energy tensor on the r.h.s. of Einstein Eqs.(14) yields (for $D=4, p=2$ ) two relations matching the coefficients in front of $\delta(\eta)$ :

$$
\left[\partial_{\eta} \widetilde{A}_{(+)}-\partial_{\eta} \widetilde{A}_{(-)}\right]_{\eta=0}=-16 \pi \chi \quad, \quad r_{0}=-\frac{a_{0}}{\pi \chi},
$$

The second relation (53) implies that the dynamical $L L$-brane tension $\chi$ must be constant (independent of the $L L$-brane proper time $\tau$ ) and it must be negative. Substituting the explicit form (51)-(52) of $\widetilde{A}_{ \pm}$in (53) gives the following expressions for the mass parameters:

$$
m_{1}=\frac{a_{0}}{2 \pi|\chi|}\left(1-\frac{4 a_{0}^{2} \beta^{2}}{3 \pi \chi^{2}}\right) \quad, \quad m_{2}=\frac{a_{0}}{2 \pi|\chi|}\left(1+\frac{8 a_{0} q^{2}}{\pi \chi^{2}}\right),
$$

as well as the relation between $a_{0}$ and $\chi$ :

$$
\chi^{2}=\frac{2 a_{0}\left(2 q^{2}+a_{0} \beta^{2}\right)}{\pi\left(1-8 a_{0}\right)} .
$$

Next, the $L L$-brane equation of motion (40), where we set $\chi=$ const, yields in the case under consideration a third matching relation at the "throat":

$$
\frac{|\chi|}{4}\left[\partial_{\eta} \widetilde{A}_{(+)}+\partial_{\eta} \widetilde{A}_{(-)}\right]_{\eta=0}+2 q^{2}-a_{0} \beta^{2}=0
$$


which upon using (54)-(55) reduces to the second relation (53), i.e., Eq.(56) does not carry any new information.

From relations (54)-(55) we conclude that all physical parameters of the asymmetric wormhole (51)-(52) are explicitly determined by the two free parameters $(q, \beta)$ - the surface electric charge density $q$ and the Kalb-Ramond charge $\beta$ of the $L L$-brane.

It remains to check that:

$$
\left.\left.\partial_{\eta} \widetilde{A}\right|_{\eta \rightarrow+0} \equiv \partial_{r} A_{(+)}\right|_{r=r_{0}}>0 \quad, \quad-\left.\left.\partial_{\eta} \widetilde{A}\right|_{\eta \rightarrow-0} \equiv \partial_{r} A_{(-)}\right|_{r=r_{0}}>0,
$$

i.e., the "throat" at $\eta=0$ must be the outer Reissner-Nordström horizon from the point of view of the "right" Reissner-Nordström "universe" $(\eta>0$, cf. (48)) and simultaneously be the inner Schwarzschild-type horizon from the point of view of the "left" Schwarzschild-de-Sitter "universe" $\eta<0$, cf. (46)). From (54)-(55) we find:

$$
\begin{array}{r}
\left.\partial_{\eta} \widetilde{A}\right|_{\eta \rightarrow+0}=\frac{\pi|\chi|}{a_{0}\left(2 q^{2}+a_{0} \beta^{2}\right)}\left[2 q^{2}\left(16 a_{0}-1\right)+a_{0} \beta^{2}\right]>0 \\
-\left.\partial_{\eta} \widetilde{A}\right|_{\eta \rightarrow-0}=\frac{\pi|\chi|}{a_{0}\left(2 q^{2}+a_{0} \beta^{2}\right)}\left[2 q^{2}+a_{0} \beta^{2}\left(16 a_{0}-1\right)\right]>0 .
\end{array}
$$

Inequalities (58)-(59) together with Eq.(55) imply the following restriction on the integration constant $a_{0}$ from the $L L$-brane dynamics (30):

$$
1 / 16<a_{0}<1 / 8 .
$$

In complete analogy one can construct another asymmetric wormhole solution where the LL-brane connects the "left" universe, which is now the exterior region of the standard Schwarzschild space-time $\left(r>r_{0}=2 m_{1}\right)$, with the "right" universe, which is the exterior region of the Reissner-Nordström-deSitter space-time $\left(r>r_{0}\right)$ beyond the outer Reissner-Nordström horizon $r=r_{0}$ :

$$
\begin{gathered}
\widetilde{A}(\eta) \equiv A_{(-)}(\widetilde{r}(\eta))=1-\frac{2 m_{1}}{\widetilde{r}(\eta)} \quad \text { for } \eta<0, \\
\widetilde{r}(\eta)=r_{0}-\eta \quad, \quad r_{0}=2 m_{1}, \\
\widetilde{A}(\eta) \equiv A_{(+)}(\widetilde{r}(\eta))=1-\frac{2 m_{2}}{\widetilde{r}(\eta)}+\frac{Q^{2}}{\widetilde{r}^{2}(\eta)}-K \widetilde{r}^{2}(\eta) \quad \text { for } \eta>0, \\
\widetilde{r}(\eta)=r_{0}+\eta \quad, \quad Q^{2} \equiv \frac{8 \pi}{a_{0}} q^{2} r_{0}^{4} \quad, \quad K \equiv \frac{4 \pi}{3} \beta^{2} .
\end{gathered}
$$

In this case the physical parameters of the asymmetric wormhole read:

$$
m_{1}=\frac{r_{0}}{2}=\frac{a_{0}}{2 \pi \chi} \quad, \quad m_{2}=\frac{a_{0}}{2 \pi \chi}\left[1+\frac{2\left(1-8 a_{0}\right)}{2 q^{2}+a_{0} \beta^{2}}\left(2 q^{2}-\frac{1}{3} a_{0} \beta^{2}\right)\right],
$$

for $\chi^{2}$ we get the same relation (55) and the same restriction (60) holds for the $L L$-brane integration constant $a_{0}$. 


\section{A Note on Einstein-Rosen "Bridge"}

In the simple special case $(q=0, \beta=0)$ the asymmetric wormhole solution (49)-(52) reduces to a symmetric wormhole solution:

$$
\begin{array}{r}
d s^{2}=-\widetilde{A}(\eta) d v^{2}+2 d v d \eta+\widetilde{r}^{2}(\eta)\left[d \theta^{2}+\sin ^{2} \theta d \varphi^{2}\right] \\
\widetilde{A}(\eta)=1-\frac{2 m}{\widetilde{r}(\eta)} \quad, \quad \widetilde{r}(\eta)=2 m+|\eta| .
\end{array}
$$

The above metric describes two identical copies of Schwarzschild exterior space-time region $(r>2 m)$, which correspond to $\eta>0$ and $\eta<0$, respectively, and which are "glued" together at the horizon $\eta=0$ (i.e., $r=2 \mathrm{~m}$ ) occupied by the LL-brane, where the latter serves as a throat of the overall wormhole solution. This is precisely the space-time manifold of the EinsteinRosen "bridge" solution in its original formulation [19] in terms of EddingtonFinkelstein coordinates. An important consequence of the present construction is that the Einstein-Rosen "bridge" wormhole does not satisfy the vacuum Einstein equations since it needs the presence of a non-trivial matter stress-energy tensor on the r.h.s. (14) which turns out to be the stress-energy tensor of a $L L$ brane (41)-(42) self-consistently derived from a well-defined reparametrizationinvariant world-volume LL-brane Lagrangian (4).

To make the connection with the original formulation [19] of the EinsteinRosen "bridge" more explicit let us recall that Einstein and Rosen start from the standard Schwarzschild metric:

$$
d s^{2}=-A(r) d t^{2}+A^{-1}(r) d r^{2}+r^{2}\left[d \theta^{2}+\sin ^{2} \theta d \varphi^{2}\right] \quad, \quad A(r)=1-\frac{2 m}{r}
$$

and introduce new radial-like coordinate $u$ by defining $u^{2}=r-2 m$, so that the metric (65) becomes:

$$
d s^{2}=-\frac{u^{2}}{u^{2}+2 m} d t^{2}+4\left(u^{2}+2 m\right) d u^{2}+\left(u^{2}+2 m\right)^{2}\left[d \theta^{2}+\sin ^{2} \theta d \varphi^{2}\right] .
$$

Then Einstein and Rosen take two identical copies of the exterior Schwarzschild space-time region $(r>2 m)$ by letting the new coordinate $u$ to vary between $-\infty$ and $+\infty$ (i.e., we have the same $r \geq 2 m$ for $\pm u$ ). The two Schwarzschild exterior space-time regions must be matched at the horizon $u=0$ (the wormhole "throat").

Let us examine whether the original Einstein-Rosen solution satisfy the vacuum Einstein equations everywhere. To this end let us consider the Levi-Civita identity (see e.g. [27]):

$$
R_{0}^{0}=-\frac{1}{\sqrt{-g_{00}}} \nabla^{2}\left(\sqrt{-g_{00}}\right)
$$

valid for any metric of the form $d s^{2}=g_{00}(r)(d t)^{2}+h_{i j}(r, \theta, \varphi) d x^{i} d x^{j}$ and where $\nabla^{2}$ is the three-dimensional Laplace-Beltrami operator $\nabla^{2}=$ 
$\frac{1}{\sqrt{h}} \frac{\partial}{\partial x^{i}}\left(\sqrt{h} h^{i j} \frac{\partial}{\partial x^{j}}\right)$. The Einstein-Rosen metric (66) solves $R_{0}^{0}=0$ for all $u \neq 0$. However, since $\sqrt{-g_{00}} \sim|u|$ as $u \rightarrow 0$ and since $\frac{\partial^{2}}{\partial u^{2}}|u|=2 \delta(u)$, Eq.(67) tells us that:

$$
R_{0}^{0} \sim \frac{1}{|u|} \delta(u) \sim \delta\left(u^{2}\right),
$$

and similarly for the scalar curvature $R \sim \frac{1}{|u|} \delta(u) \sim \delta\left(u^{2}\right)$. From (68) we conclude that:

(i) The non-vanishing r.h.s. of (68) exhibits the explicit presence of some lightlike matter source on the throat - an observation which is missing in the original formulation [19] of the Einstein-Rosen "bridge". In fact, the problem with the metric (66) satisfying the vacuum Einstein equations at $u=0$ has been noticed in ref. [19], where in Eq.(3a) the authors multiply Ricci tensor by an appropriate power of the determinant $g$ of the metric (66) vanishing at $u=0$ so as to enforce fulfillment of the vacuum Einstein equations everywhere, including at $u=0$.

(ii) The coordinate $u$ in (66) is inadequate for description of the original Einstein-Rosen "bridge" at the throat due to the ill-definiteness as distribution of the r.h.s. in (68).

As we have seen from our construction above, the proper radial-like coordinate for the Einstein-Rosen "bridge" wormhole is $\eta$ which is related to the Einstein-Rosen coordinate $u$ via non-smooth transformation:

$$
u=\operatorname{sign}(\eta) \sqrt{|\eta|} \quad, \quad \text { i.e. } u^{2}=|\eta| .
$$

Thus, we conclude that solution (64) is the proper self-consistent formulation of the original Einstein-Rosen "bridge" wormhole where the presence of LL-brane matter source at the "throat" plays crucial role for its well-definiteness.

\section{Conclusions}

I this work we have continued to explore the use of codimension-one $L L$-branes for construction of wormhole solutions of Einstein equations, in the present case - constructing asymmetric wormholes. We have strongly emphasized the crucial properties of the dynamics of $L L$-branes interacting with gravity and bulk spacetime gauge fields:

(i) The LL-brane automatically locates itself on (one of) the horizon(s) of the bulk space-time geometry ("horizon straddling");

(ii) The LL-brane tension is an additional dynamical degree of freedom unlike the case of standard Nambu-Goto $p$-branes (where it is a given ad hoc constant), and which might in particular acquire negative values;

(iii) The $L L$-brane stress-energy tensor provides the appropriate source term on the r.h.s. of Einstein equations to enable the existence of consistent non-trivial wormhole solutions;

(iv) Electrically neutral LL-branes produce symmetric wormholes, i.e., where both left and right "universes" are related via reflection symmetry and 
where, in particular, the Misner-Wheeler "charge without charge" [21] phenomenon is observed;

(v) The wormhole reflection symmetry is broken through the natural couplings of the $L L$-brane to bulk space-time gauge fields (Maxwell and 3-index Kalb-Ramond). In this way the $L L$-brane dynamically generates non-zero Coulomb field-strength in the "right" universe and non-zero cosmological constant either in the "left" or in the "right" universe which enable the existence of asymmetric (with no reflection symmetry) wormholes.

We have specifically stressed the crucial role of $L L$-branes already in the case of the "mother of all wormholes" - the classic Einstein-Rosen "bridge" manifold [19].

Finally, let us mention the crucial role of $L L$-branes in constructing nontrivial examples of non-singular black holes, i.e., solutions of Einstein equations with black hole type geometry in the bulk space-time, in particular possessing horizons, but with no space-time singularities in the center of the geometry. For further details we refer to [28], where a solution of the Einstein-Maxwell-KalbRamond system coupled to a charged $L L$-brane has been obtained describing a regular black hole. The space-time manifold of the latter consists of de Sitter interior region and exterior Reissner-Nordström region glued together along their common horizon (it is the inner horizon from the Reissner-Nordström side).

\section{Acknowledgments}

E.N. and S.P. are supported by Bulgarian NSF grant DO 02-257. Also, all of us acknowledge support of our collaboration through the exchange agreement between the Ben-Gurion University of the Negev (Beer-Sheva, Israel) and the Bulgarian Academy of Sciences.

\section{References}

[1] C. Barrabés and P. Hogan, "Singular Null-Hypersurfaces in General Relativity" (World Scientific, Singapore, 2004).

[2] K. Thorne, R. Price and D. Macdonald (Eds.), “Black Holes: The Membrane Paradigm" (Yale Univ. Press, New Haven, CT, 1986).

[3] W. Israel, Nuovo Cim. B44, 1 (1966); erratum, Nuovo Cim. B48, 463 (1967).

[4] C. Barrabés and W. Israel, Phys. Rev. D43, 1129 (1991).

[5] T. Dray and G. 't Hooft, Class. Quantum Grav. 3, 825 (1986).

[6] I. Kogan and N. Reis, Int. J. Mod. Phys. A16, 4567 (2001) [hep-th/0107163].

[7] D. Mateos and S. Ng, JHEP 0208, 005 (2002) [hep-th/0205291].

[8] J. Harvey, P. Kraus and F. Larsen, Phys. Rev. D63, 026002 (2001) [hepth/0008064];

D. Mateos, T. Mateos and P.K. Townsend, JHEP 0312 (2003) 017 [hepth/0309114];

A. Bredthauer, U. Lindström, J. Persson and L. Wulff, JHEP 0402 (2004) 051 [hepth/0401159].

[9] C. Barrabés and W. Israel, Phys. Rev. D71, 064008 (2005) [gr-qc/0502108]. 
[10] E. Guendelman, A. Kaganovich, E. Nissimov and S. Pacheva, Phys. Rev. D72, 0806011 (2005) [hep-th/0507193];

E. Guendelman, A. Kaganovich, E. Nissimov and S. Pacheva, Fortschr. der Physik 55, 579 (2007) [hep-th/0612091];

E. Guendelman, A. Kaganovich, E. Nissimov and S. Pacheva, in "Fourth Internat. School on Modern Math. Physics", eds. B. Dragovich and B. Sazdovich (Belgrade Inst. Phys. Press, Belgrade, 2007), p. 215 [hep-th/0703114];

E. Guendelman, A. Kaganovich, E. Nissimov and S. Pacheva, in "Lie Theory and Its Applications in Physics 07”, eds. V. Dobrev and H. Doebner (Heron Press, Sofia, 2008), p. 79 [arxiv:0711.1841[hep-th]].

[11] E. Guendelman, A. Kaganovich, E. Nissimov and S. Pacheva, Centr. Europ. Journ. Phys. 7, 668 (2009) [arxiv:0711.2877[hep-th]];

E. Guendelman, A. Kaganovich, E. Nissimov and S. Pacheva, in "Fifth Summer School in Modern Mathematical Physics", eds. B. Dragovich and Z. Rakic (Belgrade Inst. Phys. Press, Belgrade, 2009) [arxiv:0810.5008[hep-th]].

[12] E. Guendelman, A. Kaganovich, E. Nissimov and S. Pacheva, Phys. Lett. 673B, 288 (2009) [arxiv:0811.2882[hep-th]];

E. Guendelman, A. Kaganovich, E. Nissimov and S. Pacheva, Fortschr. der Phys. 57, 566 (2009) [arxiv:0901.4443[hep-th]].

[13] E. Guendelman, A. Kaganovich, E. Nissimov and S. Pacheva, Spherically Symmetric and Rotating Wormholes Produced by Lightlike Branes, arxiv:0904.0401[hepth], to appear in Inter. Journ. Mod. Phys. A.

[14] U. Lindström and H. Svendsen, Int. J. Mod. Phys. A16, 1347 (2001) [arxiv:hepth/0007101].

[15] W. Israel and E. Poisson, Phys. Rev. Lett. 63, 1663 (1989);

W. Israel and E. Poisson, Phys. Rev. D41, 1796 (1990).

[16] E. Guendelman, A. Kaganovich, E. Nissimov and S. Pacheva, Phys. Lett. 681B, 457 (2009) [arxiv:0904.3198[hep-th]]

[17] M. Visser, “Lorentzian Wormholes. From Einstein to Hawking” (Springer, Berlin, 1996).

[18] J. Lemos, F. Lobo and S. de Oliveira, Phys. Rev. D68, 064004 (2003) [grqc/0302049];

S. Sushkov, Phys. Rev. D71, 043520 (2005) [gr-qc/0502084];

F. Lobo, Exotic Solutions in General Relativity: Traversable Wormholes and "Warp Drive” Spacetimes, arxiv:0710.4474[gr-qc].

[19] A. Einstein and N. Rosen, Phys. Rev. 43, 73 (1935).

[20] Ch. Misner, K. Thorne and J.A. Wheeler, "Gravitation" (W.H. Freeman and Co., San Francisco, 1973).

[21] C. Misner and J. Wheeler, Ann. of Phys. 2, 525-603 (1957).

[22] A. Aurilia, H. Nicolai and P.K. Townsend, Nucl. Phys. B176, 509 (1980);

A. Aurilia, Y. Takahashi and P.K. Townsend, Phys. Lett. 95B, 265 (1980).

[23] E. Guendelman, Class. Quantum Grav. 17, 3673 (2000); Phys. Rev. D63, 046006 (2001).

[24] P. Townsend, Phys. Lett. 277B, 285 (1992);

E. Bergshoeff, L. London and P. Townsend, Class. Quantum Grav. 9, 2545 (1992) [hep-th/9206026];

J. de Azcarraga, J. Izquierdo and P. Townsend, Phys. Rev. D45, R3321 (1992).

[25] A. Eddington, Nature, 113, 192 (1924);

D. Finkelstein, Phys. Rev. D110, 965 (1958). 
[26] S. Blau, E. Guendelman and A. Guth, Phys. Rev. D35, 1747 (1987).

[27] T. Frankel, "Gravitational Curvature, An Introduction to Einstein Theory” (W.H. Freeman and Co., San Francisco, 1979).

[28] E. Guendelman, A. Kaganovich, E. Nissimov and S. Pacheva, Non-Singular Black Holes From Gravity-Matter-Brane Lagrangians, arxiv:0908.4195[hep-th], to appear in Inter. Journ. Mod. Phys. A. 